\title{
Mathematical prediction of Ying's twin universes
}

\author{
Bijan Davvaz ${ }^{1}$, Ruggero Maria Santilli ${ }^{2, *}$, Thomas Vougiouklis ${ }^{3}$ \\ ${ }^{1}$ Department of Mathematics, Yazd University, Yazd, Iran \\ ${ }^{2}$ The Institute for Basic Research, 35246 US 19 North, Palm Harbor, Florida 34684, USA \\ ${ }^{3}$ Democritus University of Trace, School of Education, 68100 Alexandroupolis, Greece
}

\author{
Email address: \\ davvaz@yazd.ac.ir(B. Davvaz), research@i-b-r.org (R. M. Santilli), tvougiou@eled.duth.gr (T. Vougiouklis)
}

\section{To cite this article:}

Bijan Davvaz, Ruggero Maria Santilli, Thomas Vougiouklis. Mathematical Prediction of Ying's Twin Universes. American Journal of Modern Physics. Special Issue: New Science Light Path on Cosmological Dark Matters. Vol. 4, No. 1-1, 2015, pp. 5-9.

doi: 10.11648 j.ajmp.s.2015040101.12

\begin{abstract}
We review Ying's twin universes, Santilli's isodual theory of antimatter, and Davvaz-Santilli-Vougiouklis two-valued hyperstructures representing matter and antimatter in two distinct but co-existing spacetimes. We identify a seemingly new map for both matter and antimatter providing a mathematical prediction of Ying's twin universes, and present a four-fold hyperstructure representing matter-antimatter as well as Ying's twin universes, all co-existing in distinct spacetimes.
\end{abstract}

Keywords: Twin Universes, Isodual, Antimatter, Hyperstructures, Spacetime

\section{Introduction}

In paper [1] and references quoted therein, Ying recalls that the 20th century laws of physics are valid for all possible conditions existing in the universe, except for the assumption of creation via the big bang conjecture which defies the conservation of energy. By applying a universal conservation law to the fundamental quantities of energy and entropy, Ying suggests that the beginning of our cosmos was divided into zero-balanced distinct universes comprising that of matter, antimatter plus two twin universes, thus maintaining conservation laws at the act of creation.

In a series of works (see monograph [2] and quoted references), Santilli proposed a new theory of antimatter which is applicable at all levels of study, from Newtonian mechanics to second quantization, with particular reference to the classical characterization of neutral antimatter bodies, such as antimatter galaxies, stars and asteroids. By recalling that charge conjugation in a Hilbert space $H$ over the field of complex numbers $C$ is characterized by the inner authomorphisms of wavefunction:

$$
\psi(t, r) \rightarrow-\psi^{\dagger}(t, r)
$$

Santilli proposed the anti-isomorphic map of all quantities for matter to the corresponding quantities for antimatter, called Santilli isoduality and denoted with the upper index ${ }^{d}$ [2].

$$
\begin{gathered}
Q(t, r, p, \psi, \ldots) \rightarrow Q^{d}\left(t^{d}, r^{d}, p^{d}, \psi^{d}, \ldots\right) \\
=-Q^{\dagger}\left(-t^{\dagger},-r^{\dagger}-p^{\dagger},-\psi^{\dagger}, \ldots\right)
\end{gathered}
$$

An important difference between maps (1) and (2) is that, for the former map, antimatter exists in our spacetime with coordinates $t, r$ while for the latter map, antimatter exists in a spacetime with coordinates $t^{d}, r^{d}$ which is completely distinct from that of matter, yet co-existing with our own spacetime so that the dimensions remains $3+1$.

Another important consequence of the isodual theory of antimatter is that the total physical characteristics of the universe are identically null under the conditions of equal amounts of matter and antimatter in the universe. This implies that the total time, total linear momentum, total energy, total entropy, etc., are identically null at all times, thus including at the time of creation, thus avoiding the violation of the conservation laws which is inherent in the big bang conjecture.

It should finally recalled that Santilli isodual theory of antimatter avoids the historical problems for antimatter moving backward in time $t^{d}=-t$ and possessing negative energy $E^{d}=-E$ thanks to the underlying novel isodual mathematics requiring the joint isoduality of physical quantities and their units of measurements. In fact, antimatter moving backward in time with negative energy referred to 
negative units of time and energy, respectively, is as causal as matter moving forward in time with positive energy referred to positive units of time and energy, respectively.

These features suggested Davvaz, Santilli and Vougiouklis to present in paper [3] a unified treatment of the matter and antimatter universes via a two-valued hyperstructure whose basic hyperunit in classical formulation is two-valued:

$$
I_{\text {tot }}=\operatorname{Diag} \cdot\left(I, I^{d}\right)
$$

where one should be aware that the symbol $I$ can represent functions, matrices, operators depending on the problem considered [2].

In this note we present, apparently for the first time, a mathematical prediction of Ying's twin universes which is independent from the conjecture of the big bang, due to the ongoing controversies, such as the return to the Middle Ages with Earth at the center of the universe which is a necessary consequence of the big bang to represent the radial character of Hubble's law $z=H d$ in all possible directions $d$ from Earth.

While studies of Ying's twin universes in a form dependent on the conjecture of the big bang are not here excluded, we believe that the derivation of Ying's twin universes in a way independent from the big bang add credibility and value toYing's hypothesis.

\section{Twin Universes}

Our mathematical prediction of Ying's twin universes is based on the simple, yet fundamental observation that map (2) from matter to antimatter is anti-Hermitean. This implies the mathematical existence of a new map, here called Ying's duality and denoted with the upper symbol ${ }^{y}$, which is merely given by the Hermitean conjugation of both the matter and antimatter universes:

$$
\begin{gathered}
Q=Q(t, r, p, \psi, \ldots) \rightarrow Q^{y}=Q^{\dagger}=Q^{y}\left(t^{y}, r^{y}, p^{y}, \psi^{y}, \ldots\right) \\
=Q^{\dagger}\left(t^{\dagger}, r^{\dagger}, p^{\dagger}, \psi^{\dagger}, \ldots\right) \\
Q^{d}=-Q^{\dagger}=-Q^{\dagger}\left(-t^{\dagger},-r^{\dagger},-p^{\dagger},-\psi^{\dagger}, \ldots\right) \rightarrow\left(Q^{d}\right)^{y} \\
=\left(-Q^{\dagger}\right)^{\dagger}=-Q(-t,-r,-p,-\psi, \ldots)
\end{gathered}
$$

thus resulting in four distinct but co-existing universes with generic quantities:

$$
\begin{gathered}
Q(t, r, p, \psi, \ldots) ;-Q^{\dagger}\left(-t^{\dagger},-r^{\dagger},-p^{\dagger},-\psi^{\dagger}, \ldots\right) ; \\
Q^{\dagger}\left(t^{\dagger}, r^{\dagger}, p^{\dagger},-\psi^{\dagger}, \ldots\right) ; \\
-Q(-t,-r,-p,-\psi, \ldots)
\end{gathered}
$$

and corresponding four-value hyperunit in classical formulation.

$$
I_{\text {tot }}=\operatorname{Diag} \cdot\left(1,1^{d}, 1^{y}, 1^{d y}\right)
$$

It should be noted that the four universes with generic quantities (6) are nontrivially distinct, as one can see for example from the underlying four distinct and incoherent
Hilbert spaces. We should also stress that spacetime remains four-dimensional for the four universes, and it is not sixteen-dimensional. Finally, we should indicate that the conjugation between one of the two Ying's universes to the other is Santilli isoduality,

$$
Q^{y} \rightarrow\left(Q^{y}\right)^{d}
$$

Consequently, in the above mathematical derivation, the total physical quantities of Ying's twin universes are identically null, of course, under the use of Santilli isodual mathematics for the transition from one universe to the other.

The hyperstructural formulation of the matter-antimatter worlds presented in [3] can be extended to a four-valued hyperstructure as follows.

The compatibility of the complexities of nature with our sensory perception has motivated the construction of multi-valued hyperstructures with hyperunits [2, 4]. In its most elementary possible formulation expressed via conventional operations, matter and antimatter can be represented via a four-valued hyperstructure characterized by the multiplicative hyperunit:

$$
E=\left\{1,1^{d}, 1^{y}, 1^{d y}\right\}
$$

where $\{\ldots\}$ represents a set, with hypernumbers:

$$
N=\left\{n, n^{d}, n^{y}, n^{d y}\right\}
$$

and related hyperproduct.

$$
\begin{gathered}
N \times M= \\
\left\{n \times m, n^{d} \times^{d} m^{d}, n^{y} \times \times^{y} m^{y}, n^{d y} \times{ }^{d y} m^{d y}\right\}
\end{gathered}
$$

where $x$ is the conventional (associative) multiplication, under which $E$ is the hyperunit for all possible hypernumbers.

The set of hypernumbers with the indicated hyperunit and hyperproduct verifies all axioms of a numerical field, thus yielding the four-valued hyperfield:

$$
F=\left\{\begin{array}{c}
F(n, \times, 1), F^{d}\left(n^{d}, \times^{d}, 1^{d}\right), F^{y}\left(n^{y}, \times^{y}, 1^{y}\right), \\
F^{d y}\left(n^{d y}, \times^{d y}, 1^{d y}\right)
\end{array}\right\}
$$

from which all remaining aspects of a four-valued hypermathematics can be constructed via known procedures. Compatibility with our sensory perception is achieved by the fact that, at the abstract realization-free level, numbers and hypernumbers, spaces and hyperspaces, etc., coincide, thus avoiding the increase of dimensionality not allowed by our sensory perception.

Algebraic hyperstructures represent a natural extension of classical algebraic structures. They were introduced in 1934 by Marty [5]. In a classical algebraic structure, the composition of two elements is an element, while in an algebraic hyperstructure, the composition of two elements is a set. Let $H$ be a non-empty set and $\times: H \times H \rightarrow P^{*}(H)$ be a hyperoperation, where $P^{*}(H)$ is the set of all non-empty subsets of $H$. The couple $(H, \times)$ is called a hypergroupoid. For any two non-empty subsets $A$ and $B$ of $H$ and $x \in H$, we define: 


$$
\begin{gathered}
A \times B=\bigcup_{a \in A, b \in B} a \times b \\
A \times x=A \times\{x\} \text { and } x \times B=\{x\} \times B
\end{gathered}
$$

A hypergroupoid $(H, \times)$ is called a semi-hypergroup if for all $a, b, c$ of $H$ we have $(a \times b) \times c=a \times(b \times c)$, which means that:

$$
\bigcup_{u \in a \times b} u \times c=\bigcup_{v \in b \times c} a \times v
$$

A hypergroupoid $(H, \times)$ is called a quasi-hypergroup if for all $a$ of $H$ we have $a \times H=H \times a=H$. This condition is also called the reproduction axiom. A hypergroupoid $(H, \times)$ which is both a semi-hypergroup and a quasi-hypergroup is called a hypergroup.

\subsection{First Proposition}

Let $(G, \cdot)$ be an abelian group and $P$ be any subset of $G$ with more than one element. We define the hyperoperation $\times_{P}$ as follows:

$$
\begin{gathered}
x \times p y= \\
\left\{\begin{array}{cc}
x \cdot P \cdot y=\{x \cdot h \cdot y \mid h & \in P\} \text { if } x \neq e \text { and } y \neq \mathrm{e} \\
x \cdot y & \text { if } x=e \text { or } y=e
\end{array}\right.
\end{gathered}
$$

We call this $P_{e}$-hyperoperation. The hyperstructure $(G, \times p)$ is a hypergroup.

A triple $(R,+, \times)$ is called a multiplicative hyperring [6] if (i) $(R,+)$ is an abelian group; (ii) $(R, \times)$ is a semi-hypergroup; (iii) for all $a, b, c \in R$, we have $a \times(b+c) \subseteq a \times b+a \times c$ and $(b$ $+c) \times a \subseteq b \times a+c \times a$; (iv) for all $a, b \in R$, we have $a \times$ $(-b)=(-a) \times b=-(a \times b)$. If in (iii) we have equalities instead of inclusions, then we say that the multiplicative hyperring is strongly distributive. Let $(\mathrm{F},+, \times)$ be a multiplicative hyperring such that:

1) $(F-\{0\}, \times)$ is a hypergroup,

2) $\times$ is strongly distributive with respect to + .

Then $(F,+, \times)$ is called a multiplicative hyperfield. For more details about hyperrings we refer to $[7,8]$.

\subsection{Second Proposition}

Let $(F,+, \cdot)$ be a field and $P$ be a non-empty subset of $(F-$ $\{0\}, \%$. Consider the hyperoperation defined in the first proposition. Then $(F,+, \times)$ is a multiplicative hyperfield.

Let $(F,+, \cdot)$ be a field and $P=\hat{I}=\left\{1,1^{d}, 1^{y}, 1^{d y}\right\}$. According to the second proposition, $(F,+, \times)$ is a multiplicative hyperfield. We define:

$$
\hat{n}=n \times \hat{I}=\left\{n, n^{d}, n^{y}, n^{d y}\right\}
$$

and we set:

$$
\widehat{F}=\{\hat{n} \mid n \in F\}
$$

We define a sum and a multiplication on $\hat{F}$ as follows:

$$
\hat{n} \oplus \widehat{m}:=\widehat{n+m} \text { and } \hat{n} \otimes \widehat{m}:=\widehat{n \cdot m}
$$

for all $n, m \in F$.

\subsection{Theorem}

$(\hat{F}, \oplus, \odot$ ) is a field and $\hat{I}$ is the unit of $\hat{F}$ (we call $\hat{I}$ a hyperunit).

Going deep in the theory of Ying's twin universes, more complicated hyperstructures can be defined as we present in the following. During last decades hyperstructures have a variety of applications in other branches of mathematics and in many other sciences. These applications range from biomathematics, conchology, inheritance, and hadronic physics or on leptons to mention but a few. The hyperstructures theory is now also widely applicable in industry and production. In books and review papers [7, 9, 10, $11,12,13,14,15]$ one can find numerous applications. $e$-hyperstructures are a special kind of hyperstructures and, in what follows, we shall see that they can be interpreted as a generalization of two important concepts for physics: Isotopies and Genotopies. On the other hand, biological systems such as cells or organisms at large are open and irreversible because they grow. The representation of more complex systems, such as neural networks, requires more advance methods, such as hyperstructures. In this manner, $e$-hyperstructures can play a significant role for the representation of complex systems in physics and biology, such as nuclear fusion, the reproduction of cells or neural systems. These applications were investigated by Santilli and Vougiouklis and later by Davvaz. Here, we review several types of $e$-hyperstructures. A hypergroupoid $(H, \cdot)$ is called an $e$-hypergroupoid if $H$ contains a scalar identity (also called unit) $e$; which means that for all $x \in H ; x \cdot e=e \cdot x=x$. In an $e$-hypergroupoid, an element $x$ ' is called inverse of a given element $x \in H$ if $e \in x \cdot x^{\prime} \cap x^{\prime} \cdot x$. Clearly, if a hypergroupoid contains a scalar unit, then it is unique, while the inverses are not necessarily unique. In what follows, we use some examples which are obtained as follows: Take a set where an operation ". " is defined, then we "enlarge" the operation putting more elements in the products of some pairs. Thus a hyperoperation " $\circ$ " can be obtained, for which we have $x \cdot y \in x^{\circ} y$, for any $x, y \in H$.

Consider the usual multiplication on the subset $\{1,-1, i,-i\}$ of complex numbers. Then, we can consider the hyperoperation ${ }^{\circ}$ defined in the following table:

$\begin{array}{lllll}\circ & 1 & -1 & i & -i \\ 1 & 1 & -1 & i & -i \\ -1 & -1 & 1 & -i & i,-i \\ i & i & -i & -1 & 1 \\ -i & -i & i & 1, i & -1, i\end{array}$

Notice that we enlarged the products $(-1) \cdot(-i),(-i) \cdot i$ and $(-i) \cdot(-i)$ by setting $(-1)^{\circ}(-i)=\{i,-i\},(-i)^{\circ} i=\{1, i\}$ and $(-i)^{\circ}(-i)=\{-1, i\}$. We obtain an $e$-hypergroupoid, with the scalar unit 1 . The inverses of the elements $-1, i,-i$ are -1 , $-i$, $i$ respectively. Moreover, the above structure is an $H_{v}$-abelian group, which means that the hyperoperation ${ }^{\circ}$ is weak associative, weak commutative and the reproductive axiom holds. 
Another kind of an $e$-hyperstructure is an $e$-hyperfield. A set $F$, endowed with an operation " + ", which we call addition and a hyperoperation, called multiplication " . ", is said to be an $e$-hyperfield if the following axioms are valid:

A) $(F,+)$ is an abelian group where 0 is the additive unit;

B) the multiplication - is weak associative;

C) the multiplication - is weak distributive with respect to + , that is, $\forall x, y, z \in F, x(y+z) \cap(x y+x z) \neq 0,(x+y) z$ $\cap(x z+y z) \neq 0$;

D) 0 is an absorbing element, that is, $\forall x \in F, 0 \cdot x=x \cdot 0=$ 0 ;

$E$ ) there exists a multiplicative scalar unit 1, that is, $\forall x \in F$, $1 \cdot x=x \cdot 1=x$

$F$ ) for every element $x \in F$ there exists an inverse $x^{-1}$, such that $1 \in x \cdot x^{-1} \cap x^{-1} \cdot x$.

The elements of an $e$-hyperfield $(F,+, \cdot)$ are called $e$-hypernumbers.

Starting with the ring $Z_{3}=\{\overline{0}, \overline{1}, \overline{2}\}$ we can obtain a hyperring by enlarging the product $\overline{2}^{\circ} \overline{2}=\{\overline{1}\}$ to $\overline{2}^{\circ} \overline{2}=$ $\{\overline{1}, \overline{2}\}$. In other words, we obtain the following table:

$\begin{array}{llll}\circ & \overline{0} & \overline{1} & \overline{2} \\ \overline{0} & \overline{0} & \overline{0} & \overline{0} \\ \overline{1} & \overline{0} & \overline{1} & \overline{2} \\ \overline{2} & \overline{0} & \overline{2} & \overline{1}, \overline{2}\end{array}$

The above structure is an $e$-hyperfield.

Let $(F,+, \cdot)$ be an $e$-hyperfield. An ordered set $a=\left(a_{1}\right.$, $\left.a_{2}, \ldots, a_{n}\right)$ of $n e$-hypernumbers of $F$ is called an $e$-hypervector and the $e$-hypernumbers $a_{i}, i \in\{1,2, \cdot, \mathrm{n}\}$ are called components of the $e$-hypervector $a$.

Two $e$-hypervectors are equals if they have equal corresponding components. The hypersums of two $e$-hypervectors $a, b$ is defined as follows:

$$
a+b=\left\{\left(c_{1}, c_{2}, \ldots, c_{n}\right) \mid c_{i} \in a_{i}+b_{i}, i \in\{1,2, \ldots, n\}\right\}
$$

The scalar hypermultiplication of an $e$-hypervector $a$ by an $e$-hypernumber $\lambda$ is defined in a usual manner:

$$
\lambda^{\circ} a=\left\{\left(c_{1}, c_{2}, \ldots, c_{n}\right) \mid c_{i} \in \lambda \cdot a_{i}, i \in\{1,2, \ldots, n\}\right\}
$$

The set $F^{n}$ of all $e$-hypervectors with elements of $F$, endowed with the hypersum and the scalar hypermultiplication is called $n$-dimensional $e$-hypervector space. The set of $m \times n$ hypermatrices is a $m n$-dimensional $e$-hypervector space.

\section{Conclusion}

We believe that, since Ying's twin universes admit a precise mathematical prediction and representation, they should be investigated in line with the ongoing research on multi-universes. An important aspect of our representation is that, while multi-universes generally require an increase of the dimensions of spacetime contrary to our sensory perception, our multi-valued representation does not, thus removing known uneasiness for increased dimensions.

We consider important the mathematical derivation of
Ying's twin universes in a way independent from the conjecture of the big bang, for the evident reason that the possible dismissal of the big bang conjecture to prevent Earth at the center of the universe should not jointly carry the dismissal of Ying's twin universes.

We finally recall the rather serious difficulties in the detection of antimatter bodies, because Santilli's isodual map (2) also applies to light, resulting in the prediction of the isodual light emitted by antimatter under which conventional telescopes are inapplicable [2], thus implying that the lack of our visual detection of antimatter, by no means, is evidence that antimatter does not exist in the large scale structure of the universe. We believe that the same rules equally apply to Ying's twin universes because, as stated by Nobel Laureate for Medicine Luc Montagnier: "Absence of proof is not proof of absence."

\section{References}

[1] L. Ying, Twin universes: universal laws of thermodynamics, American Journal of Modern Physics, vol. 4, no. 3, 2014 pp. $1-4$.

[2] R. M. Santilli, Isodual theory of antimatter with application to antigravity, Grand Unification and the spacetime machine, Springer, 2001, available for download from http://www.santilli-foundation.org/docs/santilli79.pdf.

[3] B. Davvaz, R. M. Santilli and T. Vougiouklis, Studies of multi-valued hyperstructures for the characterization of matter-antimatter systems and their extension, Proceedings of the International Conference on Lie-Admissible Formulations for Irreversible Processes, Kathmandu University, Nepal, 2011, available for download at http://www.santilli-foundation.org/hyperstructures.pdf.

[4] R. M. Santilli and T. Vougioukli, Isotopies, genotopies, hyperstructures and their applications, Proceedings of the International Workshop in Monteroduni, New Frontiers in Hyperstructures and Related Algebras, Hadronic Press, 1996, pp. 1-48.

[5] F. Marty, Sur une generalization de la notion de groupe, $8^{\text {th }}$ Congress of Mathematics in Scandenaves, Stockholm, 1934, pp. 45-49.

[6] R. Rota, Sugli iperanelli motiplicativi, Rend. Di Mat., Series VII, vol. 4, no. 2, 1982, pp. 711-724.

[7] B. Davvaz and V. Leoreanu-Fotea, Hyperring theory and applications, International Academic Press, 2007.

[8] T. Vougiouklis, Hyperstructures and their representations, Hadronic Press, Florida, 1994.

[9] P. Corsini and V. Leoreanu-Fotea, Applications of hyperstructure theory, Advances in Mathematics, Kluwer Academic Publication, Dordrecht, 2003.

[10] B. Davvaz, A brief survey of applications of generalized algebraic structures and Santilli-Vougiouklis hyperstructures, $3^{\text {rd }}$ International Conference on Lie-Admissible Treatment of Irreversible Processes (ICLATIP-3), Kathmandu University, Nepal, 2011, pp. 91-100. 
[11] B. Davvaz, R. M. Santilli and T. Vougiouklis, Studies of multi-valued hyperstructures for the characterization of matter and antimatter systems, Journal of Computational Methods in Sciences and Engineering, vol. 13, 2013, pp. 37-50.

[12] R. M. Santilli, Isonumbers and genonumbers of dimension 1, $2,4,8$, their isoduals and pseudoisoduals, and hidden numbers of dimension 3, 5, 6, 7, Algebras, Groups and Geometries, vol. 10, 1993, pp. 273-322.

[13] R. M. Santilli and T. Vougiouklis, Lie-admissible hyperalgebra,
Italian Journal of Pure and Applied Mathematics, vol. 31, 2013, pp. 239-254.

[14] T. Vougiouklis, The Santilli's theory invasion in hyperstructures, 3rd International Conference on Lie-Admissible Treatment of Irreversible Processes (ICLATIP-3), Kathmandu University, Nepal, 2011, pp.69-90.

[15] T. Vougiouklis, Some remarks on hyperstructures, Contemporary Mathematics, vol. 184, 1995, pp. 427-431. 